Acciones para la superación del Claustro de profesores de la Filial Universitaria Municipal de Jagüey Grande, Cuba. Sus resultados. Páginas 231-248 en Revista de la Escuela de Ciencias de la Educación, año 11, número 10, ENERO A DICIEMBRE DE 2015. ISSN 1851-6297. ISSN EN LÍNEA 2362-3349.

\title{
ACCIONES PARA LA SUPERACIÓN DEL CLAUSTRO DE PROFESORES DE LA FILIAL UNIVERSITARIA MUNICIPAL DE JAGÜEY GRANDE, CUBA. SUS RESULTADOS
}

Por Teresa Rodríguez Chávez*

Filial Universitaria de Jagüey Grande perteneciente a la Universidad de Matanzas

Camilo Cienfuegos, Cuba.

lisette.rodriguez@umcc.cu

Por Edith Enélida Fariñas Barrios**

Filial Universitaria de Jagüey Grande perteneciente a la Universidad de Matanzas

Camilo Cienfuegos, Cuba. edith.farinas@umcc.cu

Por Raquel Pérez Cano***

Filial Universitaria de Jagüey Grande perteneciente a la Universidad de Matanzas

Camilo Cienfuegos, Cuba. raquel.perez@umcc.cu

Recibido: 11/06/2014 Aceptado: 28/09/2014

\section{Resumen}

La educación superior en los municipios ha conllevado a la utilización de recursos tanto materiales como humanos de los territorios; la incorporación a sus claustros de profesionales de diversas ramas del saber es una oportunidad por los conocimientos que poseen de los problemas de las localidades y su experiencia profesional, pero a la vez constituye un desafío por no poseer los conocimientos pedagógicos para enfrentar la labor docente educativa. En el presente trabajo se abordan las acciones

* Máster en Ciencias de la Educación Superior. Subdirectora de Investigación y Postgrado en la Filial Universitaria de Jagüey Grande perteneciente a la Universidad de Matanzas Camilo Cienfuegos.

** Máster en Ciencias de la Educación Superior. Subdirectora docente en la Filial Universitaria de Jagüey Grande perteneciente a la Universidad de Matanzas Camilo Cienfuegos.

*** Máster en Ciencias de la Educación Superior. Directora en la Filial Universitaria de Jagüey Grande perteneciente a la Universidad de Matanzas Camilo Cienfuegos. 
Revista de la Escuela de Ciencias de la Educación, año 11, número 10, enero a diciembre de 2015. Páginas 231-248. ISSN 1851-6297. ISSN EN LINEA 2362-3349. ACCIONES PARA LA SUPERACIÓN DEL CLAUSTRO DE PROFESORES DE LA FILIAL Universitaria Municipal de Jagüey Grande, Cuba. Sus resultados. Teresa Rodríguez Chávez - Edith Enélida Fariñas Barrios - Raquel PÉrez Cano

diseñadas y ejecutadas por la Filial Universitaria Municipal de Jagüey Grande, Cuba para la superación de su claustro de profesores, desarrolladas desde sus inicios hasta la actualidad. También refiere la evolución y resultados de las mismas en cuanto a las categorías docentes, títulos académicos, socialización y comunicación de los aportes de las investigaciones, resultados del trabajo metodológico y su reflejo en la calidad de la docencia, tanto de pregrado como de posgrado. Se emplearon métodos teóricos: histórico-lógico, analítico-sintético en el estudio de la literatura científica sobre el tema, lo que facilitó la comprensión y valoración de los aspectos abordados. El método empírico de análisis de documentos posibilitó el estudio de documentos existentes sobre la temática de investigación.

\title{
Palabras clave:
}

Educación superior -Acciones - Superación de profesores-Claustro de profesores.

\section{ACTIONS FOR THE PROFESSIONAL UPGRADING OF THE TEACHERS' STAFF OF THE MUNICIPAL UNIVERSITY SITE IN JAGÜEY GRANDE, CUBA. RESULTS OBTAINED.}

\begin{abstract}
Higher education in municipalities has brought about the use of resources both material and human in the territory. The incorporation of professionals of diverse branches of knowledge, taking into account their experience, constitute a challenge due to the fact that they do not have enough pedagogical experience to face the pedagogic and educative work. Actions designed and carried out by the Municipal University Site in Jagüey Grande, Cuba are approached for the professional upgrading of the teachers' staff since previous academic courses up to the present one. The paper also refers to the evolution and results obtained in relation to the teaching categories, academic titles, socialization and communication of the results of research papers and methodological work and its reflection in the quality of teaching both undergraduate and postgraduate. Theoretical methods such as historical logical, analytical synthetical were used in the study of the scientific literature about the topic to facilitate to the comprehension and appraisal of the aspects approached. The empiric method of analysis of documents made possible the study of existing documents on the topic of research.
\end{abstract}

\section{Key words:}

Higher education - Actions - Upgrading professors - Teachers' staff.

\section{Introducción}

A partir de las transformaciones que han tenido lugar en la educación superior cubana como parte del proceso de universalización, ésta fue extendida a todo el territorio nacional con la inclusión de sus procesos sustantivos y la participación de toda la sociedad. 
Revista de la Escuela de Ciencias de la Educación, año 11, número 10, enero a diciembre de 2015. Páginas 231-248. ISSN 1851-6297. ISSN EN LINEA 2362-3349. ACCIONES PARA LA SUPERACIÓN DEL CLAUSTRO DE PROFESORES DE LA FILIAL Universitaria Municipal de Jagüey Grande, Cuba. Sus resultados. Teresa Rodriguez Chávez - Edith Enélida Fariñas Barrios - Raquel Pérez Cano

Para su cumplimiento se crearon las sedes universitarias municipales, actuales filiales, bajo el principio de utilizar los recursos disponibles en el territorio, tanto materiales como humanos; se conformaron con un claustro único integrado por profesores a tiempo completo y a tiempo parcial.

Si bien constituye una valiosa oportunidad la presencia de profesionales del territorio como profesores, dado por el conocimiento que poseen de los problemas de su localidad y la experiencia que han acumulado en su trabajo profesional que incorporan a su quehacer profesoral. Por otra parte carecen de conocimientos y experiencia pedagógica para conducir los procesos sustantivos inherentes a este nivel de enseñanza.

Como planteara Alarcón R. (2014, p.7) en la Conferencia inaugural del 9no Congreso Internacional de Educación Superior: "Las capacidades de la universidad para articularse con la sociedad de manera fructífera dependen, en gran medida, del poder cognoscitivo del que las instituciones dispongan y del modo en que sean capaces de organizarse para proyectarlo sobre las grandes necesidades sociales".

También reconoce que: "Instituciones con un profesorado no comprometido con estos conceptos y de frágil capacidad de formación e investigación, difícilmente tendrán oportunidades de abordar exitosamente su responsabilidad social. Es imprescindible que hagamos todo el esfuerzo posible por elevar al máximo el compromiso, nivel académico y científico de nuestros profesores; así como, estructurar programas de grado y posgrado, investigación e innovación que multipliquen las capacidades de nuestras universidades de producir, acumular, comprender, evaluar, diseminar y favorecer el uso social del conocimiento.

Se hace necesaria la incorporación de los profesores a la superación profesional, entendida como proceso de formación continua que transforma a los docentes con el fin de dotarlos de saberes actualizados, métodos, procedimientos y medios que les permitan desempeñarse acertadamente en su labor pedagógica, por lo que el presente trabajo tiene como objetivo: Exponer los resultados de la superación del claustro de profesores de la Filial Universitaria Municipal de Jagüey Grande.

En el desarrollo del trabajo se aplicaron métodos teóricos y empíricos. Entre los teóricos: el histórico-lógico que posibilitó un ordenamiento lógico de los contenidos abordados, análisis y síntesis en el estudio de la literatura científica sobre el tema facilitando la comprensión y valoración de los aspectos abordados. El método empírico de análisis de documentos posibilitó el estudio de documentos existentes sobre la temática de investigación.

\section{Desarrollo}

La universidad como institución en la que se aprende el conjunto de saberes permitirá al futuro profesional iniciarse en el ejercicio de una profesión 
Revista de la Escuela de Ciencias de la Educación, año 11, número 10, enero a diciembre de 2015. Páginas 231-248. ISSN 1851-6297. ISSN EN LINEA 2362-3349. ACCIONES PARA LA SUPERACIÓN DEL CLAUSTRO DE PROFESORES DE LA FILIAL Universitaria Municipal de Jagüey Grande, Cuba. Sus resultados. Teresa Rodriguez Chávez - Edith Enélida Fariñas Barrios - Raquel Pérez Cano

con eficiencia y avanzar en los conocimientos de un ámbito del saber, de forma que pueda emprender la investigación y profundización de los mismos en su vida profesional, al mismo tiempo también es un espacio de aprendizaje de carácter humano y ético.

La enseñanza en Cuba ha sido revolucionada en el presente siglo, acorde con las características del mundo actual, donde se manejan términos como "sociedad de la información", "sociedad del conocimiento múltiple", "sociedad del aprendizaje continuo" que exigen a las instituciones universitarias convertirse en centros de educación permanente de donde egresen estudiantes con una preparación apreciable para vivir en sociedad y les viabilice la adaptación a este mundo versátil. Insta formar profesionales capaces de comprender la esencia del proceso de construcción de los conocimientos, cuyo volumen y calidad crece, cambia y se complejiza cada día más.

Dentro de este contexto un concepto significativo lo constituye el proceso de universalización de la educación superior y la más trascendental conquista consiste en llevar los estudios superiores a todos los municipios del país con la creación de las sedes universitarias municipales, actuales filiales universitarias municipales. Acercar la universidad al lugar de residencia o laboral de las personas significa crear facilidades para alcanzar el pleno acceso a la educación terciaria. Para cumplir los propósitos del pleno acceso y que el estudiante pueda combinar su formación como universitario con la laboral, la puesta en práctica de la modalidad semipresencial es esencial.

En la educación superior cubana, la semipresencialidad se considera como "la modalidad pedagógica que posibilita el amplio acceso y la continuidad de estudios de todos los ciudadanos, a través de un proceso de formación integral, enfatizando más en los aspectos que el estudiante debe asumir por sí mismo; flexible y estructurado; en el que se combina el empleo intensivo de los medios de enseñanza con las ayudas pedagógicas que brindan los profesores; adaptable en intensidad a los requerimientos de éstos y a los recursos tecnológicos disponibles para llevarla a cabo"(MES, 2006, p.3).

La adopción de la modalidad semipresencial demanda actitudes pedagógicas diferentes a las tradicionales de la enseñanza presencial, dirigidas a garantizar un aprendizaje óptimo del estudiante, capaz de asumir de modo activo su propio proceso de formación con la guía, el apoyo y compañía de los profesores. La semipresencialidad en la educación superior cubana posee características a considerar que la diferencian de la presencialidad y exigen el reconocimiento de fundamentos psicopedagógicos adaptados a ella, entre los que se pueden mencionar: (Ibid)

- Formación integral, con mayor énfasis en la actividad independiente del estudiante, para que sea capaz de asumir de modo activo su proceso de formación integral. 
Revista de la Escuela de Ciencias de la Educación, año 11, número 10, enero a diciembre de 2015. Páginas 231-248. ISSN 1851-6297. ISSN EN LINEA 2362-3349. ACCIONES PARA LA SUPERACIÓN DEL CLAUSTRO DE PROFESORES DE LA FILIAL Universitaria Municipal de Jagüey Grande, Cuba. Sus resultados. Teresa Rodriguez Chávez - Edith Enélida Fariñas Barrios - Raquel Pérez Cano

- Amplio y progresivo empleo de los medios de enseñanza y las tecnologías educativas, que posibiliten el aprendizaje independiente del estudiante y compensen las actividades de las clases de la modalidad presencial, las que el profesor no puede realizar por el limitado tiempo de contacto con sus estudiantes.

- Desarrollo de adecuados niveles de motivación en los estudiantes.

- Atención a las necesidades educativas grupales.

- Utilización de las potencialidades de todos los escenarios educativos en función de garantizar la calidad de la formación integral del estudiante.

- Trabajo colaborativo en red desde el nivel nacional hasta los escenarios educativos en la base que garantice la integralidad y coherencia de los diferentes elementos que conforman la modalidad de estudios semipresencial.

- Impacto social, no sólo en cuanto a la trascendencia intrínseca que tiene la universalización de la educación superior para el desarrollo de la sociedad, sino además por las potencialidades de la modalidad de estudio para incidir en el desarrollo local sostenible y en la propia formación de los estudiantes.

Ginoris, O. (2006, p. 3) plantea criterios pedagógicos y didácticos a tener en consideración en esta modalidad otorgando al proceso de enseñanza aprendizaje más carácter formativo que informativo, donde el estudiante asuma una actividad responsable, capaz de buscar y producir información, concediéndole tanta importancia a la cognición como a la metacognición, orientado a la autorregulación del aprendizaje. Las características planteadas exigen la superación continua que garantice la preparación eficiente del profesor.

Es necesario un proceso de formación que no se base solamente en el componente cognitivo, al respecto González V. y González R.M. (2008, p.187) afirman que es de gran importancia: "la formación humanística de profesionales competentes y comprometidos con el desarrollo social"; también refieren que: "ocurre a partir de la integración de conocimientos, habilidades, motivos y valores que se expresan en un desempeño profesional eficiente, ético y de compromiso social". Ello se logra cuando junto a los contenidos propios de las disciplinas y asignaturas, el profesor propicia el desarrollo del pensamiento crítico, de la reflexión y el interés cognoscitivo.

Es reconocido de manera generalizada por diversos autores la importancia del personal docente para conducir el proceso docente educativo, sobre ello Piña N. (2004, p. 56) plantea que varios organismos internacionales han considerado que: "los docentes son el recurso más vital para promover la modernización y las normas superiores; su contribución, formación, distribución y concesión de incentivos apropiados son claves para cualquier sistema educativo eficaz". 
Revista de la Escuela de Ciencias de la Educación, año 11, número 10, enero a diciembre de 2015. Páginas 231-248. ISSN 1851-6297. ISSN EN LINEA 2362-3349. ACCIONES PARA LA SUPERACIÓN DEL CLAUSTRO DE PROFESORES DE LA FILIAL Universitaria Municipal de Jagüey Grande, Cuba. Sus resultados. Teresa Rodriguez Chávez - Edith Enélida Fariñas Barrios - Raquel Pérez Cano

Es tal la importancia atribuida a los docentes y a su superación que la misma se convierte en prioridad para varios países, además es abordada por organismos internacionales como la Organización de Naciones Unidas para la Educación, la Ciencia y la Cultura (UNESCO) y la Organización Regional de Educación Superior en América Latina (CRESAL).

En la Conferencia Mundial sobre la Educación Superior de la UNESCO en París (1998, 2009), fueron aprobaron documentos que explicitan la necesidad de la educación permanente del profesorado universitario y de su formación docente. Se plantea: "Como la educación a lo largo de toda la vida exige que el personal docente actualice y mejore sus capacidades didácticas y sus métodos de enseñanza... es necesario establecer estructuras, mecanismos y programas adecuados de formación docente". Además se considera que: "Un elemento esencial para las instituciones de la enseñanza superior es una enérgica política de formación docente del personal" (UNESCO, 2009).

En Cuba la superación de su personal docente reviste vital importancia para lograr elevar a planos superiores tanto el desarrollo profesional como humano y al lograrlo se contribuye a la transformación de la sociedad por la incidencia del profesor en la formación del profesional que conducirá en un futuro no muy lejano los procesos productivos y las transformaciones sociales de la sociedad. Por ello se hace necesario encauzar de manera planificada, organizada y en consonancia con las necesidades de los profesores la superación que posibilitará su desempeño de manera acertada.

Varios autores han abordado la temática, entre ellos se encuentran: Añorga J. (1994), Díaz M. (1996), Bernaza G. (2004), Fraga O. (2005), Castro O.J. (2012), García X. y Rivera O. (2012), Morales M. y Carballo M. (2012), García A. (2013) los que reconocen a la superación profesional como proceso de formación que permite a graduados universitarios la ampliación y profundización de conocimientos de manera que puedan lograr un mejor desempeño profesional y así elevar su contribución a la sociedad en la que se insertan.

Añorga J. (1994, p. 23) define a la superación profesional como un "Conjunto de procesos de enseñanza-aprendizaje que posibilita a los graduados universitarios la adquisición y el perfeccionamiento continuo de los conocimientos y habilidades requeridas para un mejor desempeño de sus responsabilidades y funciones laborales."

Se asume en el presente trabajo por corresponder con los objetivos propuestos la definición de superación del claustro de profesores que plantea Díaz M. (1996): "un proceso de formación continua a lo largo de toda su vida profesional, que produce un cambio y mejora las conductas docentes en las formas de pensar, valorar y actuar como docentes" (citado por Fraga, 2005, p.15).

Se concuerda con García A. (2013) al afirmar que con el perfeccionamiento profesional se asciende al desarrollo social, el cual exige de procesos continuos de creación, difusión, transferencia, adaptación y aplicación de conocimientos. 
Revista de la Escuela de Ciencias de la Educación, año 11, número 10, enero a diciembre de 2015. Páginas 231-248. ISSN 1851-6297. ISSN EN LINEA 2362-3349. ACCIONES PARA LA SUPERACIÓN DEL CLAUSTRO DE PROFESORES DE LA FILIAL Universitaria Municipal de Jagüey Grande, Cuba. Sus resultados. Teresa Rodriguez Chávez - Edith Enélida Fariñas Barrios - Raquel Pérez Cano

También al plantear que el saber, estrechamente vinculado a la práctica, es una fuerza social transformadora que se debe fomentar permanentemente para promover el desarrollo sostenible de la sociedad. Lo anterior explica que el perfeccionamiento organizativo del proceso de superación de los profesores parta de la concepción del docente como un profesional que requiere elevar su preparación para el desarrollo de la docencia.

En Cuba como afirma Román (2014) la formación continua del profesorado se perfecciona a través de la conjugación armónica y sistémica de la educación de postgrado, la investigación pedagógica y la preparación metodológica. Los resultados a través de dicho proceso permiten al docente universitario la obtención de categorías docentes básicas y superiores, así como títulos académicos y científicos que los van identificando cada vez más como profesores universitarios. El trabajo metodológico antes mencionado permite una preparación, seguimiento y control de su actividad didáctica en la clase.

La educación de posgrado es: "una de las direcciones principales de trabajo de la educación superior en Cuba, y el nivel más alto del sistema de educación superior, dirigido a promover la educación permanente de los graduados universitarios. En la educación de posgrado concurren uno o más procesos formativos y de desarrollo, no solo de enseñanza-aprendizaje, sino también de investigación, innovación, creación artística y otros, articulados armónicamente en una propuesta docente-educativa pertinente a este nivel" (MES, 2004, p. 2).

En el artículo 8 del citado Reglamento se plantea que:

La educación de posgrado enfatiza el trabajo colectivo y la integración en redes, a la par que atiende de modo personalizado las necesidades de formación de los estudiantes de este nivel; promueve la superación continua de los graduados universitarios, el desarrollo de la investigación, la tecnología, la cultura y el arte. Para cumplir esta variedad de funciones, la educación de posgrado se estructura en superación profesional y formación académica" (MES, 2004, p.3).

Los profesores tienen como responsabilidad resolver científicamente los problemas que se presentan en su labor pedagógica para dirigir eficientemente el proceso docente educativo y contribuir a la vez a la formación del profesional capaz de transformar y perfeccionar la sociedad en la que vive, por lo cual la investigación pedagógica alcanza relevancia dentro de su quehacer profesional.

Se comparte el planteamiento de De Armas al referir que: "Cuando la actividad investigativa pasa a formar parte de la actividad profesional pedagógica del docente este desarrolla una actitud reflexiva, creativa que le permite acometer el perfeccionamiento de la actividad docente educativa en el aula, la efectividad en el trabajo metodológico y de autopreparación generando expe- 
Revista de la Escuela de Ciencias de la Educación, año 11, número 10, enero a diciembre de 2015. Páginas 231-248. ISSN 1851-6297. ISSN EN LINEA 2362-3349. ACCIONES PARA LA SUPERACIÓN DEL CLAUSTRO DE PROFESORES DE LA FILIAL Universitaria Municipal de Jagüey Grande, Cuba. Sus resultados. Teresa Rodriguez Chávez - Edith Enélida Fariñas Barrios - Raquel Pérez Cano

riencias y aportes de diversos niveles de novedad y originalidad" (De Armas, s/a, p. 1).

El trabajo metodológico según el artículo 24 del Reglamento para el Trabajo Docente y Metodológico en la educación superior es:

(...) la labor que, apoyados en la Didáctica, realizan los sujetos que intervienen en el proceso docente educativo, con el propósito de alcanzar óptimos resultados en dicho proceso, jerarquizando la labor educativa desde la instrucción, para satisfacer plenamente los objetivos formulados en los planes de estudio" (MES, 2007,p.5).

El Ministerio de Educación Superior (MES) reconoce la necesidad de la superación sistemática de los profesores y la considera como una tarea de gran importancia y necesidad para preservar, enriquecer y potenciar los logros de ese nivel educacional. Para encauzar el trabajo en este sentido estableció el Sistema de Superación de Profesores de los Centros de Educación Superior adscriptos al MES con la Instrucción 3/2008.

Se consideran como principios que regulan el Sistema a:

1.- Superación continua, integral y pertinente: Superación a lo largo de la vida profesional, que abarca todos los ámbitos de actuación del profesor y de acuerdo con sus necesidades de superación.

2.- Carácter integrador: A partir de las prioridades en la formación político ideológica, pedagógica, profesional e investigativa de los profesores.

3.- Múltiple: Combina las diferentes formas organizativas del posgrado y otras con el objetivo de lograr una formación integral y continua de los profesores.

4.- Flexible: Articulado de tal manera que permita al profesor ir venciendo los diferentes objetivos de formación de acuerdo con sus potencialidades y condiciones y ritmo de estudio.

5.- Pleno acceso: Todos los profesores pueden participar en todas las actividades que necesiten del sistema.

6.- Basado en la motivación, el aprendizaje colaborativo y la autogestión del aprendizaje: A partir de la comprensión de la necesidad de superación de cada profesor y en colaboración con los demás.

7.- Esfuerzo adicional: Basado en la motivación de los profesores, requiere un esfuerzo adicional de los mismos, en particular, de aquellos a tiempo parcial.

8.- Delimitación de responsabilidades: La dirección del CES establece las obligaciones de las diferentes instancias de la Sede Central y las que pueden cumplir las Sedes Universitarias Municipales con sus propios recursos humanos.

10.- Autoevaluación: como vía para la determinación de necesidades propias de superación de cada profesor, esencial para la proyección de acciones 
Revista de la Escuela de Ciencias de la Educación, año 11, número 10, enero a diciembre de 2015. Páginas 231-248. ISSN 1851-6297. ISSN EN LINEA 2362-3349. ACCIONES PARA LA SUPERACIÓN DEL CLAUSTRO DE PROFESORES DE LA FILIAL Universitaria Municipal de Jagüey Grande, Cuba. Sus resultados. Teresa Rodriguez Chávez - Edith Enélida Fariñas Barrios - Raquel Pérez Cano

de superación profesional o formación académica en el SSP (MES, 2008, p. 2).

Acerca de la importancia de la superación de los profesores para su desempeño en la formación de profesionales que respondan a las necesidades de la sociedad Alarcón R. refiere que:

(...) Las capacidades de la universidad para articularse con la sociedad de manera fructífera dependen, en gran medida, del poder cognoscitivo del que las instituciones dispongan y del modo en que sean capaces de organizarse para proyectarlo sobre las grandes necesidades sociales. También afirma que: "Instituciones con un profesorado no comprometido con estos conceptos y de frágil capacidad de formación e investigación, difícilmente tendrán oportunidades de abordar exitosamente su responsabilidad social. Es imprescindible que hagamos todo el esfuerzo posible por elevar al máximo el compromiso, nivel académico y científico de nuestros profesores; así como, estructurar programas de grado y posgrado, investigación e innovación que multipliquen las capacidades de nuestras universidades de producir, acumular, comprender, evaluar, diseminar y favorecer el uso social del conocimiento (Alarcón, 2014, p.7).

En la Filial Universitaria Municipal de Jagüey Grande se incorporan como profesores universitarios a tiempo parcial profesionales que se desempeñan en el territorio en sus respectivas ramas del saber, poseedores de vasta experiencia y resultados relevantes; pero carecen de formación pedagógica, por lo que fue necesario a partir del diagnóstico de sus necesidades diseñar y ejecutar acciones de superación tanto de carácter colectivo como individual y así contribuir a su formación docente.

La Filial Universitaria Municipal de Jagüey Grande ha planificado y organizado acciones de superación del claustro de profesores atendiendo a 3 direcciones:

- Dirección 1: Superación de posgrado.

- Determinación y desarrollo de actividades de posgrado que contribuyan al perfeccionamiento de su formación docente, científica y política.

$\checkmark$ Incorporación a programas para la obtención de títulos académicos desarrollados por la Sede Central:

Maestría en Administración de Empresas

Maestría en Ciencias de la Educación Superior

Programa de Posgrado en Red de la Educación Superior

Especialidad en Trabajo Social

$\sim$ Doctorado Curricular Colaborativo en Ciencias Pedagógicas 
Revista de la Escuela de Ciencias de la Educación, año 11, número 10, enero a diciembre de 2015. Páginas 231-248. ISSN 1851-6297. ISSN EN LINEA 2362-3349. ACCIONES PARA LA SUPERACIÓN DEL CLAUSTRO DE PROFESORES DE LA FILIAL Universitaria Municipal de Jagüey Grande, Cuba. Sus resultados. Teresa Rodriguez Chávez - Edith Enélida Fariñas Barrios - Raquel Pérez Cano

Diseño y ejecución de cursos de posgrado (superación profesional):

Introducción a los Fundamentos de la Nueva Universidad Cubana

La educación en valores a través de la instrucción en la modalidad semipresencial

Metodología de la investigación

$\sim$ Problemas sociales de la ciencia y la tecnología

$\sim$ Curso de Inglés

$\sim$ El artículo científico

- Dirección 2: Investigación pedagógica.

- Diagnóstico de necesidades y carencias de los profesores, de sus potencialidades profesionales, pedagógicas y didácticas para contribuir al perfeccionamiento de su formación como profesores universitarios.

- Estudio y debate de los planes de estudio de las carreras para determinar de forma cooperada el enfoque interdisciplinario de los contenidos y problemas de la profesión que contribuya a entrenar a los profesores para desarrollar la actividad investigativa.

- Estimulación de la búsqueda de posibles temas de investigación teóricos o empíricos, a partir de la valoración de problemas docentes y de la especialidad.

- Desarrollo de investigaciones sobre el resultado de su quehacer pedagógico y profesional.

- Intercambio y exposición de los resultados investigativos.

- Generalización de los resultados científicos.

- Dirección 3: Trabajo metodológico.

- Diseño de la Estrategia y del Plan metodológico de la FUM y de las carreras, de forma integrada para lograr el trabajo conjunto de profesores en el perfeccionamiento de su labor docente, a partir de las necesidades de superación que poseen.

- Diseño de estrategias metodológicas, pedagógicas y didácticas que le permitan desarrollar la docencia en correspondencia con las necesidades sociales y las particularidades del modelo pedagógico.

- Ejecución de clases metodológicas, abiertas y de comprobación que contribuyen al perfeccionamiento de la labor educativa desde la instrucción en la modalidad semipresencial.

- Incorporación al trabajo metodológico del aprendizaje cooperativo en la solución a preguntas y problemas propios de la profesión pertinentes al contexto local. 
Revista de la Escuela de Ciencias de la Educación, año 11, número 10, enero a diciembre de 2015. Páginas 231-248. ISSN 1851-6297. ISSN EN LINEA 2362-3349. ACCIONES PARA LA SUPERACIÓN DEL CLAUSTRO DE PROFESORES DE LA FILIAL Universitaria Municipal de Jagüey Grande, Cuba. Sus resultados. Teresa Rodriguez Chávez - Edith Enélida Fariñas Barrios - Raquel Pérez Cano

- Ejecución de actividades que estimulen la autorreflexión y autovaloración de los profesores sobre sus puntos más fuertes y más débiles acerca de su desarrollo profesional.

- Control a la calidad del proceso docente educativo donde se constate el desempeño a partir de la superación.

- Evaluación del desempeño alcanzado por el profesor como resultado de la superación, donde se estimula la autovaloración, autocontrol y autoevaluación.

Las acciones ejecutadas en función del desarrollo profesional y humano del claustro de profesores a través de la superación posgraduada, la investigación educativa y el trabajo metodológico han posibilitado el perfeccionamiento de su desempeño en la formación de profesionales revolucionarios, cultos, creativos y competentes, al respecto Betto F. plantea que: "El primer deber del educador no es formar mano de obra especializada o calificada para el mercado de trabajo. Es formar seres humanos felices, dignos, dotados de conciencia crítica, participantes activos en el desafío permanente de perfeccionar el socialismo, que considero que es el nombre político del amor" (Betto, 2014, p.7).

En la FUM al comenzar cada curso escolar se realiza el diagnóstico de las necesidades de la formación humanística del claustro, la preparación pedagógica, la superación profesional de posgrado, la formación para la investigación, el empleo de las tecnologías de la información y las comunicaciones (TIC) en los procesos universitarios, el conocimiento de idioma inglés; la preparación en los problemas sociales de la ciencia, la tecnología y la sociedad; y otros aspectos relevantes para la superación del profesorado.

A partir de las necesidades se diseña el Sistema de Superación de Profesores, el cual es analizado con los coordinadores de carrera y profesores, se elabora el Plan de superación de los profesores a tiempo parcial y se incluyen las tareas de superación en el Plan de trabajo del profesor propio en correspondencia con sus necesidades y carencias.

El cumplimiento del plan de superación de los profesores a tiempo parcial y el plan de trabajo del profesor propio son controlados sistemáticamente, a través de los resultados de los posgrados -los que constan en actas-, de la presentación de los resultados científicos en eventos y las publicaciones, de la participación en actividades metodológicas y de los resultados de los controles a actividades docentes.

Con sistematicidad mensual se efectúa reunión de análisis y control del trabajo donde se valoran los resultados de la etapa según lo planificado, se ejecutan balances parciales y al concluir el primer semestre y el curso escolar se incorpora el estado de cumplimiento de la superación en la evaluación profesoral. 
Revista de la Escuela de Ciencias de la Educación, año 11, número 10, enero a diciembre de 2015. Páginas 231-248. ISSN 1851-6297. ISSN EN LINEA 2362-3349. ACCIONES PARA LA SUPERACIÓN DEL CLAUSTRO DE PROFESORES DE LA FILIAL Universitaria Municipal de Jagüey Grande, Cuba. Sus resultados. Teresa Rodríguez Chávez - Edith Enélida Fariñas Barrios - Raquel Pérez Cano

Con el diseño, ejecución y control de las acciones de superación del claustro de profesores se evidencian avances significativos en: la composición por categorías docentes, títulos académicos, participación en eventos científicos, publicaciones, resultados de controles de actividades docentes, los que se expresan a continuación:

Se analiza el Informe de la Evaluación institucional y las Fichas del centro de los cursos escolares 2007-2008, 2011-2012 y 2013-2014 (primer semestre) para comparar el comportamiento de las categorías docentes de los profesores a tiempo completo (PTC) y a tiempo parcial (PTP). Al comenzar la Sede Universitaria Municipal en el 2002-2003 existía un profesor a tiempo completo que era instructor y 20 PTP, de ellos 19 instructores y 1 asistente, ninguno poseía título académico. A partir del desarrollo de las acciones de superación se aprecian avances significativos en cuanto a la categoría docente de los profesores tanto a tiempo completo como a tiempo parcial como se refleja en los gráficos 1 y 2. En la actualidad son profesores auxiliares el $50 \%$ de los PTC y el $36 \%$ de los PTP, son instructores el $7 \%$ de los PTC y el $28 \%$ de los PTP.

En cuanto al título académico también ha habido progreso, son másteres o especialistas el $64,3 \%$ de los PTC y el $56 \%$ de los PTP, además son doctores el $6 \%$ de éstos. Gráficos 3 y 4

\section{Categoría docente. Profesores a tiempo completo.}

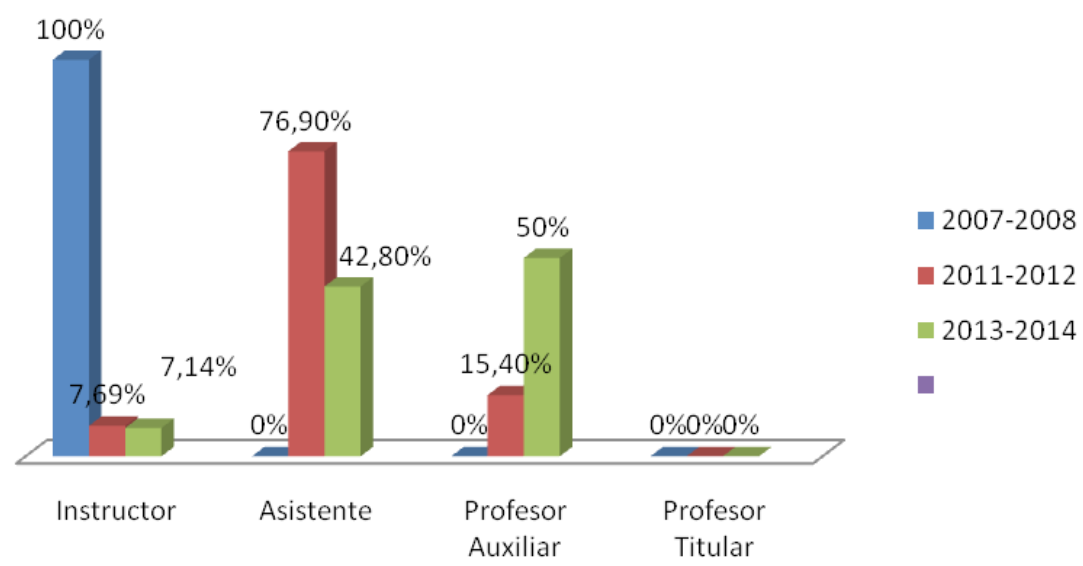

Gráfico 1. Fuente: Elaboración propia basada en Fichas del centro. Estadísticas. Ministerio de Educación Superior. 
Revista de la Escuela de Ciencias de la Educación, año 11, número 10, enero a diciembre de 2015. Páginas 231-248. ISSN 1851-6297. ISSN EN LINEA 2362-3349. ACCIONES PARA LA SUPERACIÓN DEL CLAUSTRO DE PROFESORES DE LA FILIAL Universitaria Municipal de Jagüey Grande, Cuba. Sus resultados. Teresa Rodriguez Chávez - Edith Enélida Fariñas Barrios - Raquel Pérez Cano

Categoría docente. Profesores a tiempo parcial.

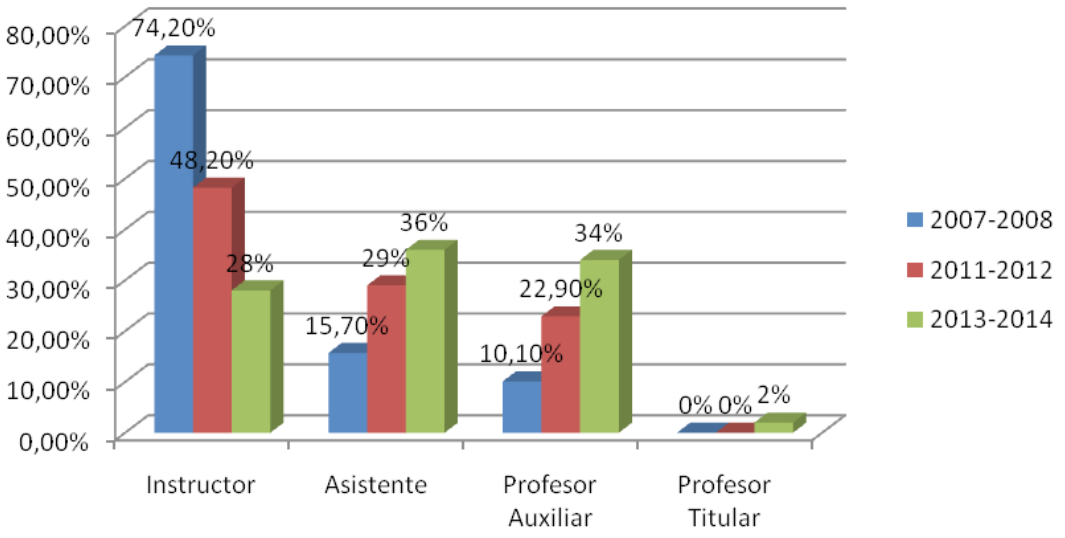

Gráfico 2. Fuente: Elaboración propia basada en Fichas del centro. Estadísticas. Ministerio de Educación Superior.

Título académico. Profesores a tiempo completo.

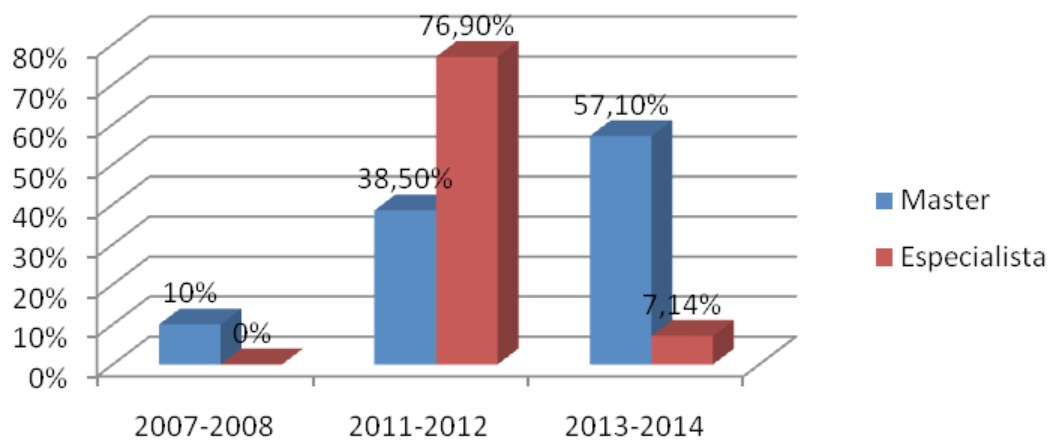

Gráfico 3. Fuente: Elaboración propia basada en Fichas del centro. Estadísticas. Ministerio de Educación Superior. 
Revista de la Escuela de Ciencias de la Educación, año 11, número 10, enero a diciembre de 2015. Páginas 231-248. ISSN 1851-6297. ISSN EN LINEA 2362-3349. ACCIONES PARA LA SUPERACIÓN DEL CLAUSTRO DE PROFESORES DE LA FILIAL Universitaria Municipal de Jagüey Grande, Cuba. Sus resultados. Teresa Rodríguez Chávez - Edith Enélida Fariñas Barrios - Raquel PÉrez Cano

Título académico y grado científico. Profesores a tiempo parcial.

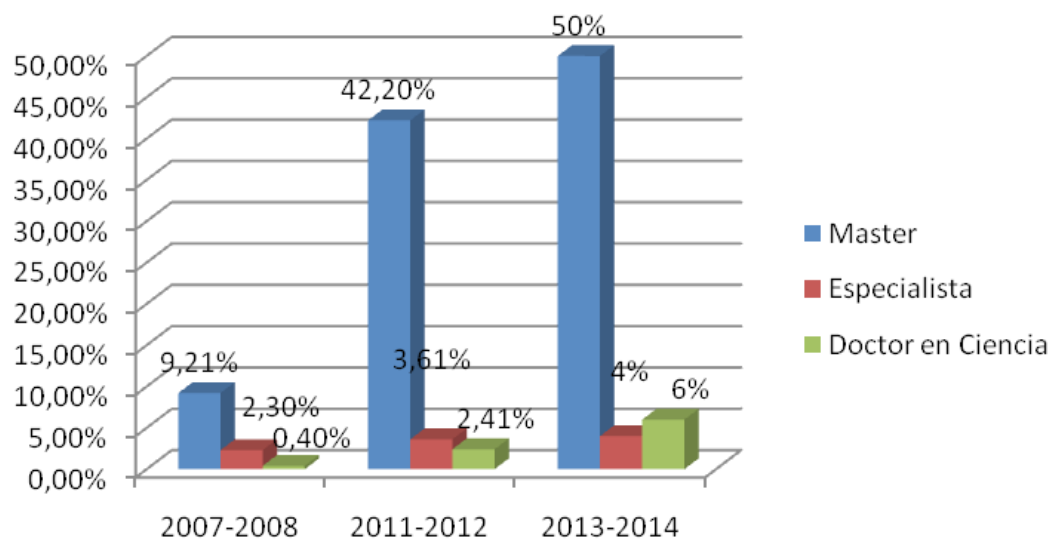

Gráfico 4. Fuente: Elaboración propia basada en Fichas del centro. Estadísticas. Ministerio de Educación Superior.

La superación del claustro de profesores en cuanto a la formación académica posibilita su mejor desempeño en las actividades profesionales e investigativas. En la actualidad cursan estudios de doctorado tres PTC y dos PTP; cursan maestrías tres PTC y siete PTP; la incorporación de profesores a esta forma de posgrado contribuye al cambio y mejora tanto de su desempeño como docentes e investigadores como a la contribución al desarrollo del territorio por el aporte de sus resultados científicos.

La formación posgraduada incluye a los cursos de posgrados como superación profesional, tiene el objetivo de la formación permanente y la actualización sistemática de los graduados universitarios, el perfeccionamiento del desempeño de sus actividades profesionales y académicas (MES, 2004, p.3). Han cursado y aprobado los cursos concebidos como parte de su Plan de superación un número significativo de profesores: Introducción a los Fundamentos de la Nueva Universidad Cubana 656 profesionales del territorio, cifra elevada por ser parte de la preparación para categorizarse como profesor universitario en el territorio, Metodología de la investigación 189, Problemas Sociales de la Ciencia y la Tecnología 151, Inglés 45, El artículo científico 75 y La educación desde la instrucción en la modalidad semipresencial 31.

Como reconoce Alarcón R.: "es la universidad la institución mejor preparada para gestionar el conocimiento y aplicarlo en la formación de grado y posgrado, investigación e innovación aportando a la solución de necesidades sociales, culturales, económicas y ambientales. Gestionar conocimiento y conectarlo con 
Revista de la Escuela de Ciencias de la Educación, año 11, número 10, enero a diciembre de 2015. Páginas 231-248. ISSN 1851-6297. ISSN EN LINEA 2362-3349. ACCIONES PARA LA SUPERACIÓN DEL CLAUSTRO DE PROFESORES DE LA FILIAL Universitaria Municipal de Jagüey Grande, Cuba. Sus resultados. Teresa Rodriguez Chávez - Edith Enélida Fariñas Barrios - Raquel Pérez Cano

la sociedad es uno de los pilares del conocimiento". (Alarcón, 2014, p.7) Los resultados de la producción de conocimientos resulta importante compartirlos, socializarlos, por lo que reviste importancia que los profesores presenten los mismos en eventos y obtengan visibilidad a través de las publicaciones.

En el territorio se organizan Seminarios Científicos Metodológicos con carácter anual donde los profesores presentan trabajos relacionados con las prioridades, orientaciones metodológicas y resultados docentes y científico metodológico y con carácter bienal los eventos municipales correspondientes a los Congresos Internacionales de Universidad. En ellos se han presentado desde el evento del 2008 a la actualidad 86 ponencias de las cuales han sido premiadas 43 , ha sido importante la participación en los eventos de carácter provincial y han participado en los Congresos Internacionales siete profesores de la FUM. También ha sido notable la participación en la Convención Internacional de la Universidad de Matanzas desde los años 2007 hasta el 2013 con 20 trabajos. Se registra la participación de 130 ponencias en diversos eventos.

Además de las publicaciones en los eventos de carácter provincial e internacional también se han logrado 100 en monografías desde los años 2008 hasta la actualidad, en revistas de los grupos 3 y 4 se han publicado tres artículos y en libro "La Nueva Universidad Cubana" tres artículos de profesores de la FUM, se obtuvieron tres registros Cenda (dos de ellos informáticos) y en el año 2009 se obtuvo el Premio Citma territorial con el trabajo: Sistema de Educación Ambiental de Jagüey Grande. En Fórum de Ciencia y Técnica Provincial se obtuvo un premio y un estudiante tutorado por profesora de la FUM fue ganador del Concurso Nacional de Computación en la categoría de estudiantes que usan la computación como apoyo en la solución de problemas.

El trabajo metodológico es la labor que, apoyados en la didáctica, realizan los sujetos que intervienen en el proceso docente educativo, con el propósito de alcanzar óptimos resultados en dicho proceso, jerarquizando la labor educativa desde la instrucción, para satisfacer plenamente los objetivos formulados en los planes de estudio (MES, 2007, p.14).

El profesor es el responsable fundamental de que la asignatura que imparte posea la calidad requerida, desarrollando una labor educativa desde la instrucción. Para ello debe poseer una adecuada preparación pedagógica y dominar los contenidos de la asignatura; así como orientar, controlar y evaluar a los estudiantes para lograr un adecuado dominio de dichos contenidos, en correspondencia con los objetivos generales de la asignatura, contribuyendo así a su formación integral.

Como parte del trabajo docente metodológico se ha planificado y ejecutado en cada curso escolar el sistema de actividades metodológicas que han constituido espacios y alternativas para el intercambio sistemático de saberes y experiencias en temas tales como:la estructura metodológica de la clase encuentro, la formación de valores en la modalidad semipresencial, la interdisciplinariedad 
Revista de la Escuela de Ciencias de la Educación, año 11, número 10, enero a diCIembre de 2015. PÁginas 231-248. ISSN 1851-6297. ISSN EN LINEA 2362-3349. ACCIONES PARA LA SUPERACIÓN DEL CLAUSTRO DE PROFESORES DE LA FILIAL Universitaria Municipal de Jagüey Grande, Cuba. Sus resultados. Teresa Rodríguez Chávez - Edith Enélida Fariñas Barrios - Raquel PÉrez Cano

y la transdisciplinariedad, el trabajo con las estrategias formativas, con énfasis en la preparación de asignaturas y labor educativa desde la instrucción. Estas actividades han contribuido a mejorar el desempeño pedagógico y didáctico de los profesores para la conducción del proceso docente educativo, evidenciado en los resultados de los controles a las actividades docentes realizados tanto por la Sede Central como a nivel de FUM y carrera.

La superación desarrollada a los profesores en la FUM ha posibilitado que el personal docente actualice sus conocimientos en sus respectivas ramas del saber, desarrolle capacidades pedagógicas, se encuentre mejor preparado para darle respuesta a las problemáticas tanto docentes como de su actividad profesional y contribuya a formar profesionales con conocimiento de su realidad, con desarrollo del pensamiento crítico y con ello dar respuesta a las exigencias sociales de la época.

\section{Conclusiones}

- La superación pedagógica, metodológica y científica de los profesores de la educación superior cubana en los municipios reviste vital importancia al posibilitar la incorporación de saberes y experiencias de profesionales de diversas especialidades en los territorios al contribuir a la apropiación y desarrollo de conocimientos y habilidades necesarios para conducir el proceso de formación de profesionales.

- Las acciones de superación del claustro de profesores de la Filial Universitaria Municipal de Jagüey Grande diseñadas y ejecutadas teniendo en cuenta las necesidades y demandas de los profesores posibilitarían la presencia de profesores más calificados en cuanto a sus categorías docentes, títulos académicos, mejor desempeño en su actividad docente educativa así como avances en la comunicación y visibilidad de sus resultados investigativos.

\section{Referencias bibliográficas}

- $\quad$ Alarcón Ortiz, R. (2014). Universidad socialmente responsable. Conferencia inaugural 9no Congreso Internacional Universidad 2014.

- $\quad$ Añorga Morales J., Lazo J. (1994). Proyecto para la Educación del sistema de Educación Avanzada. UASB. Sucre, Bolivia

- Betto, F. (2014). El papel del educador en la formación política de los educandos. Conferencia inaugural 9no Congreso Internacional Universidad 2014. Editorial Félix Varela.

- Castro Escarrá, O.J. (2012). Una concepción sistémica para la superación del personal docente del Ministerio de Educación. [CD-ROM]. La Habana: Evento Universidad 2012, IV Taller internacional: La formación universitaria de profesionales de la educación.

- De Armas, N. (s/a). "A modo de introducción: los resultados científicos como aporte de la investigación educativa". En: Colectivo de autores. Los resultados científicos como aporte de la investigación educativa. Universidad Pedagógica "Félix Varela". 
Revista de la Escuela de Ciencias de la Educación, año 11, número 10, enero a diciembre de 2015. PÁginas 231-248. ISSN 1851-6297. ISSN EN LINEA 2362-3349. ACCIONES PARA LA SUPERACIÓN DEL CLAUSTRO DE PROFESORES DE LA FILIAL Universitaria Municipal de Jagüey Grande, Cuba. Sus resultados. Teresa Rodríguez Chávez - Edith Enélida Fariñas Barrios - Raquel Pérez Cano

- $\quad$ Fraga Luque, O. (2005). Estrategia de superación profesional para la preparación teórica metodológica del docente en el contenido de la ética martiana. Tesis presentada en opción al grado de Doctor en Ciencias Pedagógicas, U. P. "Félix Varela", Villa Clara.

- García, M. A. (2013). Concepción desarrolladora del proceso de superación profesional del docente. En: Atlante. Cuadernos de Educación y Desarrollo, agosto 2013, Disponible en http://atlante.eumed.net/superacion-profesional

- García Navarro, X. Y Rivera O. (2012). "La Universidad de Ciencias Pedagógicas, su papel en la superación de los docentes de la Educación Especial". [CD-ROM]. La Habana: Evento Universidad 2012, IV Taller internacional La formación universitaria de profesionales de la educación.

- Ginoris Quesada, O. (2006). La semipresencialidad y sus exigencias pedagógicas y didácticas en la sede universitaria municipal, [En CD-ROM]. Fundamentos Didácticos de la Nueva Universidad Cubana. Universidad de Matanzas Camilo Cienfuegos, Cuba.

- González Maura, V; y González Tirados, R.M. (2008). Competencias genéricas y formación profesional: un análisis desde la docencia universitaria. Revista iberoamericana de educación. $N^{\circ}$ 47, p. 185-209

- MES(2004). Reglamento Educación de Posgrado. República de Cuba. Resolución No. 132/2004. La Habana.

- $\quad$ MES (2006). La modalidad semipresencial. Versión final aprobada por el consejo de dirección del MES. La Habana: MES.

- MES (2007). Reglamento para el Trabajo Docente y Metodológico en la educación superior. Resolución NO. 210/2007

- MES (2008). Sistema de superación de profesores (SSP) de los centros de educación superior adscritos al Ministerio de Educación Superior. INSTRUCCIÓN No. 3 /08

- Morales González, M. y Carballo Barco M. (2012). La superación profesional del tutor para el desarrollo de la actividad científica ante los actuales retos de la Educación Superior. [CD-ROM]. La Habana: Evento Universidad 2012, IV Taller internacional "La formación universitaria de profesionales de la educación"

- Piña Tovar, N. (2004). Estrategia de Superación desde visiones de Educación Permanente para los profesores de Educación Física de Saltillo, Coahuila, México. Tesis doctoral. ISCF "Manuel Fajardo", Villa Clara.

- Román Cao, E. (2014). Papel de la pedagogía en la universidad contemporánea. El profesor especialista. Memorias 9no Congreso Internacional Universidad 2014. PED-003

- UNESCO (2009). Conferencia Mundial de Educación Superior. Compendio de la Declaración Mundial sobre la Educación Superior. Las Nuevas Dinámicas de la Educación Superior y de la Investigación para el Cambio Social y el Desarrollo. Paris, 5-8 de julio de 2009. [on line] [consultado 16 de abril 2012]. Disponible en: http://www.unesco.org/ new/es/education/resources/online-materials/publications/ 
